\title{
FEVER THERAPY AT DAYTON, OHIO
}

By AMBROSE J. KING, F.R.C.S., Chief Assistant Medical Officer, L.C.C. (Whitechapel) Clinic

THE treatment of venereal diseases with fever induced by physical means has been the subject of a considerable and increasing number of reports in medical literature during the past few years. Most of these reports have come from the United States and the large majority have described results which are satisfactory and often remarkable. Clinic medical officers, and others who treat venereal diseases in this country, have not been slow to recognise the possibilities of fever therapy and must frequently regret their inability to apply this method to those of their cases which do not respond to the ordinary measures. Up to the present, native conservatism and difficulties as regards apparatus and personnel have combined to delay the introduction of this treatment here. As time passes and fever therapy becomes an established procedure in other countries, it is increasingly evident that these difficulties must be overcome and hyperpyrexia must receive the necessary study and trial in our own clinics and hospitals.

The reports indicate that good results are obtained by a number of different types of apparatus ; and it seems to be generally agreed that the method of raising the temperature is not of primary importance provided that due regard is paid to the safety of the patient, the effectiveness of the apparatus as a pyretogenic agent and the comfort of the patient-in that order of importance. The skill and judgment of medical and nursing care before, during and after treatment, are the factors to which the greatest attention must be paid.

The paper which Dr. Walter Simpson, Director of the Kettering Institute of Medical Research at Dayton, Ohio, read before the Medical Society for the Study of Venereal Diseases in May, I936,1 has focussed the interest of members of the Society and readers of this Journal on one 


\section{BRITISH JOURNAL OF VENEREAL DISEASES}

of these forms of apparatus, the air-conditioning unit known as the Kettering hypertherm, which was devised and elaborated at the Kettering Institute, and is now in constant use in twenty-four of the leading hospitals in the United States.

The following is a brief account of impressions received during a visit to the parent Institute at Dayton, Ohio, from April 8th to May Ist this year. From considerations of space, I have omitted any description of the preliminary investigation and nursing technique, and also of the apparatus, which, although it has been modified, does not differ in principle from that described by Dr. Simpson last year. During this visit I saw 88 treatments given, and, for the large majority, I was present during the whole period of induction, maintenance and cooling.

To record some general impressions : it seemed to me that the greatest praise was due to the efficiency, enthusiasm and esprit de corps of the personnel of the department. It was quite clear that the great success which this team of workers has achieved in obtaining therapeutic results, in avoiding casualties in the experimental stage and in training doctors and nurses from other centres, has been due to these outstanding qualities. The work is heavy and exacting; the nursing technique involves physical strain combined with constant watchfulness and anxiety; but these difficulties are happily and effectively surmounted by the constant devotion and loyalty of all the staff. It is possible that the dramatic improvement which so frequently results from treatment is in some measure responsible for this admirable attitude. It was encouraging to see that the enthusiasm of the staff for the treatment was fully shared by many of the patients who could speak of little else than the remarkable results which had been achieved in their own particular cases. At the time of my visit the medical staff of this department were compelled to spend much of their time in tactfully refusing treatment to the very large number of patients with whom they were unable to deal owing to limitations of time, staff and accommodation.

It was clear that this treatment is strenuous and involves the patient in considerable discomfort and stress. It should never be undertaken lightly and, as many of the workers in this field have emphasised, 268 


\section{FEVER THERAPY AT DAYTON, OHIO}

patients should be selected and prepared just as carefully as for major surgical operations. The after treatment is equally important. The amount of discomfort and apprehension which the patient experiences is largely determined by the nervous stability and psychological outlook of the individual concerned ; but, in the majority of cases such difficulties are limited to the induction period. Once the required temperature level is reached, the discomforts are much lessened and fears are allayed. The patient is helped through this difficult stage by small doses of sedatives, but even more by the constant sympathy and encouragement of the skilful nursetechnician. It was obvious at once that a female nurse is essential for this work and that she must be the possessor of outstanding qualities. During the period of maintenance of fever the patients chiefly complain of weariness due to prolonged inactivity, combined with some physical exhaustion in the later stages. Nevertheless during this time, relieved of discomfort and helped by sedatives, they frequently sleep or drowse for considerable periods. Delirium is seen occasionally in those of nervous temperament, and for this reason it is essential that each hypertherm should be accommodated in a separate room. During my stay, no patient demanded that the treatment should be terminated; nor was it discontinued for any other reason. At no time were further sessions of fever refused after experience of the treatment, but it frequently happened that patients were so impressed with the results that they returned enthusiastically for more. I saw no burns or any other harmful results of treatment.

As is customary with doctors and nurses who study in this department, I was given the opportunity of personal experience of the induction period of fever without the aid of sedatives. In my own case the temperature was raised to $104^{\circ} \mathrm{F}$. in the space of one hour and ten minutes, and the pulse rate did not rise above II6 beats per minute. The period of induction was certainly uncomfortable but by no means unbearable. There is no doubt that the average patient would gladly submit to this in the hope of obtaining rapid relief from symptoms. My condition after this experience was not comparable with that of a patient who has endured a prolonged period of maintenance of fever, but as soon as my temperature 
returned to normal I was able to resume ordinary activities without any ill-effects.

In general it may be said that the type of patient who is most welcomed and most encouraged to attend for fever therapy in this department is the severely complicated or neglected case which is the result of the failure or faulty application of the ordinary methods of treatment. During my period of study every attempt was made to enable me to see the effects of fever on the various manifestations of syphilis and gonorrhœa. I was able to examine the following patients-amongst others with non-venereal conditions - and to follow the effects of fever in each case :-

3 cases of gonococcal arthritis.

I case of gonococcal epididymitis, vasitis, prostatitis and seminal vesiculitis.

I case of gonococcal urethritis, anterior and posterior.

I case of gonococcal iritis. This patient had arthritis also and is included above.

I case of gonococcal cervicitis.

2 cases of interstitial keratitis due to pre-natal syphilis.

I case of primary syphilitic chancre of the penis.

I case of primary syphilitic chancre of the penis with secondary lesions of the skin and mucous membranes.

I case of syphilitic choroiditis.

I case of oldstanding syphilitic osteomyelitis of tibia.

4 cases of latent syphilis with serum reactions which had proved irreversible by chemotherapy alone.

Some brief notes on the progress of these patients may be of interest.

Arthritis.-One patient had recent acute arthritis of the wrist secondary to gonococcal urethritis. This was the most acutely and severely inflamed gonococcal joint that I have ever seen. Edema extended from fingers to elbow, and was accompanied by some acute superficial lymphangitis. No movement of the joint was possible and any attempt at manipulation was extremely painful. The patient's temperature varied from IoI $^{\circ} \mathrm{F}$. to $\operatorname{IO2}^{\circ} \mathrm{F}$ : and he complained of constant severe pain. He was treated by five hours of fever at $105^{\circ} \mathrm{F}$. to $106^{\circ} \mathrm{F}$. 


\section{FEVER THERAPY AT DAYTON, OHIO}

followed by three-day intervals by two further treatments of eight hours at $106^{\circ} \mathrm{F}$. to $107^{\circ} \mathrm{F}$. The pain was relieved as soon as the temperature was raised during the first treatment. The following day some degree of movement was possible, and although there was some slight recurrence of pain it was of minor degree. The subsequent sessions of fever removed all the signs of inflammation. At the end of treatment there was some wasting of the forearm muscles and a certain amount of stiffness of the joint. Nevertheless, movements were full and free from pain, and there was no doubt that with use and exercise full function would rapidly be restored. At the same time all the signs and symptoms of urethritis disappeared, and I was able to massage the prostate on the day following the last treatment, the specimen expressed showing no excess of leucocytes. On rectal examination, I found no evidence of acute inflammatory change in the prostate or seminal vesicles, and there was no "flare-up" of the joint condition as the result of massage.

The second case of arthritis was that of a patient with gonococcal urethritis and multiple arthritis of the lower limbs involving both knee joints and one ankle joint. He had been bedridden for some weeks and was unable to walk. There was much peri-articular infiltration of the affected joints and gross wasting of the muscles above and below the knees. He improved rapidly under treatment, and after the fourth session of fever was able to walk a short distance without the aid of crutches. All signs of the active infective process had disappeared. Naturally, in such a case it would take some weeks of massage and movements to restore the condition of the joints and muscles to normal.

The third case of arthritis was also of the multiple type and had reached the chronic stage. The patient had also gonococcal iritis of one eye. There was noticeable improvement in both conditions after five hours of fever at $105^{\circ} \mathrm{F}$. to $106^{\circ} \mathrm{F}$. This patient began treatment at the end of my period of study and I was unable to follow his case further.

- Gonococcal Infections other than Arthritis.-The patient with acute epididymitis, vasitis, prostatitis and seminal vesiculitis was free from all pain after one session of fever and clinically cured after three sessions at three-day intervals. Some residual thickening of the affected 
organs remained, but I was able to massage the prostate and seminal vesicles on the day after the last treatment without any untoward result. The resulting specimen was reported to show no microscopic evidence of infection.

The patient with uncomplicated urethritis was apparently free from infection after three treatments occupying five days. After the second treatment there were no signs apart from a slight haziness of the first specimen of urine, but the third treatment was given as a precautionary measure. Again I was able to massage the prostate on the day following the last treatment, and there was no evidence of residual infection.

The patient with gonococcal cervicitis had only two treatments before my departure, but was making good progress as judged by the clinical appearances.

Syphilitic Infections.-All the patients with syphilis had chemotherapy combined with fever in accordance with a scheme of treatment which consisted of fifty hours of fever at $105^{\circ} \mathrm{F}$. to $106^{\circ} \mathrm{F}$. given weekly in five-hour sessions during a period of ten weeks and combined on each occasion with intravenous injections of neoarsphenamine 0.3 grams and bismuth salicylate 0.2 grams intramuscularly. The injections were then continued weekly for twenty weeks without sessions of fever.

Both patients with interstitial keratitis made excellent progress under treatment but the individual variation of response of these conditions to chemotherapy is so great that it was not possible to draw any conclusions.

I formed the opinion that the superficial lesions in the two cases of early syphilis cleared up more rapidly in response to the combined treatment than is the case with chemotherapy alone. The secondary lesions had practically disappeared within two days. I was unable to follow the serological response since the blood specimens were sent away to Dr. Kahn's laboratory and there was some delay before the series of results was received. Study of the notes of the four patients with latent serofast syphilis, and of other similar patients whose treatment I did not see, made it clear that in the majority of cases fever plus chemotherapy caused a steady decline in the number of positive units in the Kahn quantitative tests.

The patient with choroiditis attended for treatment on 
one occasion only. He had had a course of treatment previously and was in the habit of returning for an occasional fever session. He was a highly intelligent man, and was convinced that this treatment had arrested progressive loss of sight and had effected some restoration of vision.

The patient with syphilitic osteomyelitis of the tibia was a debilitated subject and advanced in years. Caution was essential and it was considered advisable to give short occasional sessions of fever. His condition had been most neglected and secondary infection was a prominent feature. Chemotherapy alone had proved ineffective, but with the addition of fever there was slow but definite improvement in the ulcerative condition of the legs.

Many "follow-up" patients visited the Kettering Institute during my visit there, and a number of these assured me that fever had cured their various conditions when all other means had failed.

Nothing approaching a searching analysis of the effect of fever therapy was possible in the short time available, and these brief notes record no more than a few impressions. Nevertheless, this evidence is presented in the hope that it may serve to emphasise conclusions which may be drawn from the literature. It is clear that in the treatment of the acute complications of gonorrhœa results can be achieved by this method which cannot be obtained with the forms of treatment now at our disposal. Almost equally striking results have been recorded in the treatment of uncomplicated gonorrhœa. If only for the treatment of gonorrhœa, fever therapy must command its place.

As regards the treatment of syphilis, the position cannot be so clearly stated. Leading syphilogists in the United States view with suspicion the introduction of physically induced fever for the treatment of syphilis. They regard as premature and dangerous any claims that the combination of fever with chemotherapy will shorten the duration of necessary treatment in early syphilis, or that physically induced fever may serve as an adequate substitute for malarial therapy in the treatment of neurosyphilis. At the Kettering Institute no such claims are made, but these points are under investigation. It is fully realised that years must pass before the first question 
BRITISH JOURNAL OF VENEREAL DISEASES

can be answered, and that as regards the second both methods have their indications. Physically induced fever seeks to supplement and not to supplant malarial therapy.

\section{REFERENCE}

(I) Simpson, W. S.: Brit. J. Ven. Dis., 1936, 12, p. 133. 